\title{
Influence of Permeability and Injection Orientation Variations on Dispersion Coefficient during Enhanced Gas Recovery by $\mathrm{CO}_{2}$ Injection
}

\author{
Muhammad Kabir Abba *, Athari Al-Otaibi, Abubakar Jibrin Abbas, Ghasem Ghavami Nasr and \\ Martin Burby
}

Petroleum and Gas Research Group, The University of Salford, Manchester M4 5WT, UK; A.al-otaibi@edu.salford.ac.uk (A.A.-O.); a.j.abbas@salford.ac.uk (A.J.A.); g.g.nasr@salford.ac.uk (G.G.N.); m.burby@salford.ac.uk (M.B.)

* Correspondence: m.k.abba1@salford.ac.uk

Received: 5 May 2019; Accepted: 14 June 2019; Published: 18 June 2019

\begin{abstract}
This investigation was carried out to highlight the influence of the variation of permeability of the porous media with respect to the injection orientations during enhanced gas recovery (EGR) by $\mathrm{CO}_{2}$ injection using different core samples of different petrophysical properties. The laboratory investigation was performed using core flooding technique at $1300 \mathrm{psig}$ and $50{ }^{\circ} \mathrm{C}$. The injection rates were expressed in terms of the interstitial velocities to give an indication of its magnitude and variation based on the petrophysical properties of each core sample tested. Bandera Grey, Grey Berea, and Buff Berea sandstone core samples were used with measured permeabilities of 16.08, 217.04, and $560.63 \mathrm{md}$, respectively. The dispersion coefficient was observed to increase with a decrease in permeability, with Bandera Grey having the highest dispersion coefficient and invariably higher mixing between the injected $\mathrm{CO}_{2}$ and the nascent $\mathrm{CH}_{4}$. Furthermore, this dispersion was more pronounced in the horizontal injection orientation compared to the vertical orientation with, again, the lowest permeability having a higher dispersion coefficient in the horizontal orientation by about $50 \%$. This study highlights the importance of the permeability variation in the design of the injection strategy of EGR and provides a revision of the $\mathrm{CO}_{2}$ plume propagation at reservoir conditions during injection.
\end{abstract}

Keywords: enhanced gas recovery; longitudinal dispersion coefficient; injection orientation; supercritical $\mathrm{CO}_{2} ; \mathrm{CO}_{2}$ permeability

\section{Introduction}

The effects of greenhouse gas (GHG) emissions in the form of global warming with the resulting subsequent climate change cannot be overemphasized. The authors of [1] reported that the most significant of the GHG emissions are carbon dioxide $\left(\mathrm{CO}_{2}\right)$, methane $\left(\mathrm{CH}_{4}\right)$, dinitrogen oxide $\left(\mathrm{N}_{2} \mathrm{O}\right)$, and other gases. Amongst all these gases, they iterated that $\mathrm{CO}_{2}$ makes up about $76 \%$ of the total global emissions of GHGs. Incidentally, combustion of fossil fuels is the central source of the global $\mathrm{CO}_{2}$ emissions and the oil and gas industry alone accounts for $65 \%$ of global $\mathrm{CO}_{2}$ emissions [2]. Furthermore, $\mathrm{CO}_{2}$ emissions from the oil and gas industry is ever increasing as a result of the high energy demand and at a rate of $1.7 \%$ per annum between the 1990s and early 2000s, and at an even higher rate of 3.1\% per annum between 2000 and 2010 [3]. Therefore, the environmental consequences associated with $\mathrm{CO}_{2}$ emissions have forced researchers in the oil and gas industry to come up with technologies to curb the proliferation of anthropogenic $\mathrm{CO}_{2}$ due to the oil and gas activities. An avenue with a growing potential to address this issue is by the injection of the $\mathrm{CO}_{2}$ emissions into geological 
formations like oil and gas reservoirs [4] using enhanced oil/gas recovery processes. Enhanced gas recovery (EGR) by $\mathrm{CO}_{2}$ injection and sequestration is a simultaneous process whereby $\mathrm{CO}_{2}$ is injected into a natural gas $\left(\mathrm{CH}_{4}\right)$ reservoir to displace $\mathrm{CH}_{4}$ and store $\mathrm{CO}_{2}$ in the reservoir.

EGR is still in its pilot/test phase and has not been widely accepted due to the nature of the gas-gas displacement whereby $\mathrm{CO}_{2}$ disperses into $\mathrm{CH}_{4}$ during the process and the recovered $\mathrm{CH}_{4}$ will be heavily contaminated with the injected $\mathrm{CO}_{2}$. This will affect the market value of the recovered $\mathrm{CH}_{4}$ given that one of the reasons for the choice of $\mathrm{CH}_{4}$ reservoirs as potential storage sites is that the recovered natural gas will offset part of the cost of the sequestration process [5]. Therefore, efforts are being made to stall the incessant mixing during EGR, and this is only achievable when the physics of the mixing are better understood. Pivotal to the adoption of the technique is understanding the mechanisms and factors which influence the interaction between the gases in situ, which leads to mixing at reservoir conditions. This will eventually provide an avenue to characterise gas systems for better injection scenarios and explore the potential and the viability of EGR as an adopted method of $\mathrm{CO}_{2}$ sequestration.

In our previous works [6-9], we studied some of the factors that affect in situ mixing between the injected $\mathrm{CO}_{2}$ and the displaced $\mathrm{CH}_{4}$ which have not been previously considered. These factors include the connate water salinity, the injection orientation and, also, the flow behaviour of $\mathrm{CO}_{2}$ in its supercritical state during EGR displacement. This research, however, focuses on the effect of injection orientation of the $\mathrm{CO}_{2}$ on the dispersion of the gas in consolidated sandstone cores with an emphasis on permeability variation. The injection orientation determines the path of the $\mathrm{CO}_{2}$ plume propagation through the pore spaces of the reservoir rock, which is also very important when it comes to the dip angle between the injector and producer wells. Consistent with our previous results, horizontal injections showed that the segregation of $\mathrm{CO}_{2}$ to the bottom of the core sample-due to its higher density of supercritical $\mathrm{CO}_{2}$ compared to that of $\mathrm{CH}_{4}$ (showed in Figure 1)-led to higher residence time of the $\mathrm{CO}_{2}$ traversing the length of the core sample, thereby increasing $\mathrm{CO}_{2}$ dispersion. Invariably, dispersion in the vertical injection orientation of the $\mathrm{CO}_{2}$ was significantly less than that in the horizontal orientation.

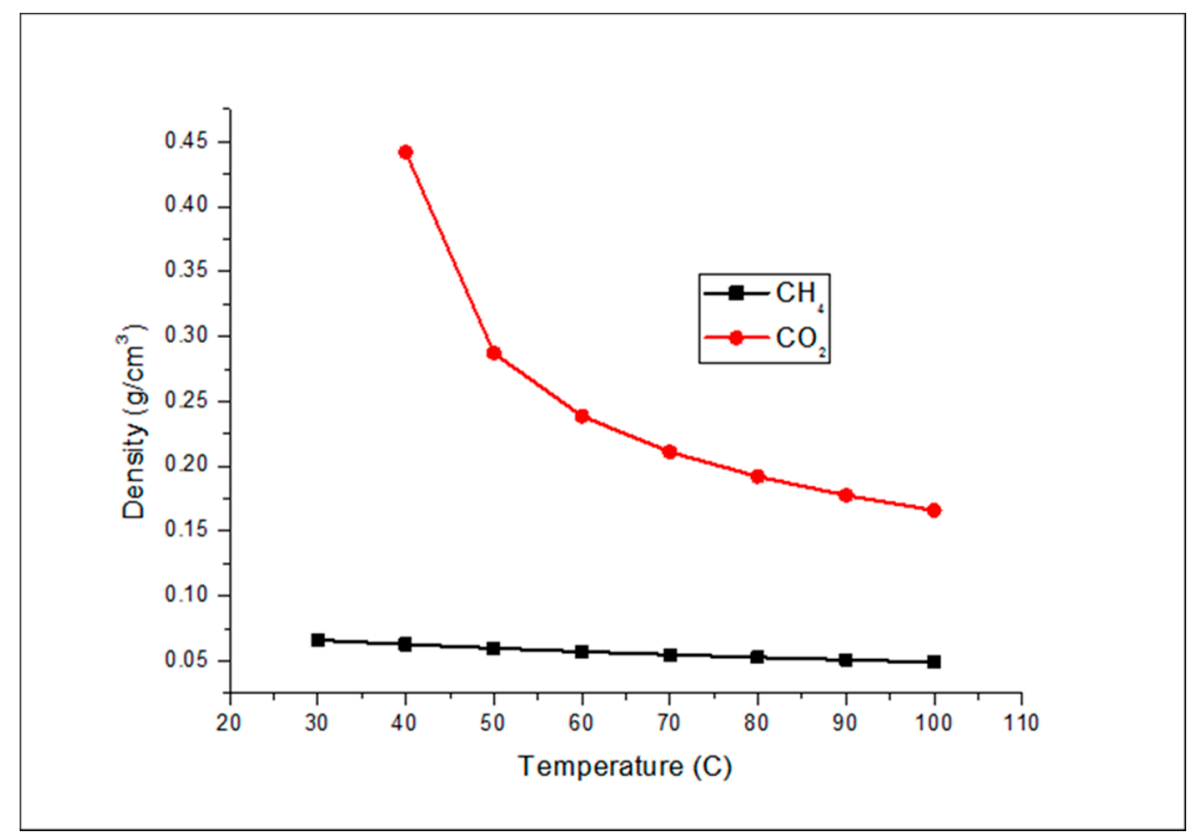

Figure 1. $\mathrm{CH}_{4}$ and $\mathrm{CO}_{2}$ density as a function of temperature at 1300 psi.

The authors of [10] carried out a horizontal dispersion of $\mathrm{CO}_{2}$ in $\mathrm{CH}_{4}$ in sand packs as the porous medium and also concluded that gravity plays a significant role in the dispersion of $\mathrm{CO}_{2}$ during displacement applications. Their study included long and short sand packs with similar permeabilities, 
while here, different consolidated core samples with different petrophysical properties and equal length were employed to ascertain the effects of their variation on dispersion. The reasoning behind the use of equal length core samples was to minimise systemic disparities in our measurements. Given that permeability is a function of length, the pore structure variation which is responsible for the permeability difference between the core sample was explored, and the interplay between the core sample and the gases was evaluated. Therefore, the variation of the injection orientation and permeability was analysed to showcase their influence on dispersion coefficient.

\section{Materials and Methods}

The core samples were obtained from Kocurek Industries USA whose petrophysical properties are shown in Table 1. Gas permeability was obtained using a core flooding and the porosity was evaluated using Helium Porosimetry. Research grade $\mathrm{CO}_{2}$ and $\mathrm{CH}_{4}$ were obtained from BOC UK both with purity of $>99.999 \%$.

Table 1. Dimensions and petrophysical properties of core samples.

\begin{tabular}{ccccc}
\hline Core Samples & Length $(\mathbf{m m})$ & Diameter $(\mathbf{m m})$ & ${ }^{*}$ Porosity $(\%)$ & ${ }^{*}$ Permeability $(\mathbf{m d})$ \\
\hline Grey Berea & 76.27 & 25.22 & $19-20$ & $200-315$ \\
Bandera Grey & 76.00 & 25.47 & 21 & 30 \\
Buff Berea & 76.18 & 24.95 & 26 & $350-600$ \\
\hline
\end{tabular}

* Properties provided by suppliers.

Apparatus and Procedure

(1) Porosity and permeability measurements

The Helium Porosimeter PORG 2007M (Corelab, OK, USA and Gas Permeater PERG $200^{\mathrm{TM}}$ (Corelab, OK, USA) were used to measure the porosity and permeability of the core samples respectively. Details of the equipment description, principles of operation, and procedure can be found in our previous works [8]. The results are shown in Table 2.

Table 2. Measured petrophysical properties.

\begin{tabular}{ccc}
\hline Core Samples & Porosimetry Porosity (\%) & Measured Permeability (md) \\
\hline Bandera Grey & 17 & 16.08 \\
Grey Berea & 20 & 217.04 \\
Buff Berea & 26.27 & 560.63 \\
\hline
\end{tabular}

(2) Core flooding

Core flooding equipment, also from CoreLab Oklahoma, UFS 200 (details are presented in [7]), was used in this work. It simulated the displacement of $\mathrm{CH}_{4}$ by supercritical $\mathrm{CO}_{2}$ at reservoir conditions. The core holder was originally horizontally orientated; however, a stand was constructed to change this orientation to vertical in order to vary the injection orientation. The same procedure was adopted as the one employed in [9], the only difference being in the using different core samples of different permeabilities and porosities. The schematics of the setup are shown in Figure 2. After carrying out the same experiments at the same conditions in the horizontal orientation for each core sample, the core holder was installed vertically, and the same experiments were repeated using the same conditions as in the previous horizontal experiments.

Injection was made from the bottom of the core holder in the vertical orientation. The injection orientation adopted in this study was not based on the dip angle of injection during the flooding and recovery processes. The experiments were not field scale depictions of the injection strategy, rather, we were looking at core to pore scale investigation of the flow physics of $\mathrm{CO}_{2}$ plume as it displaces nascent $\mathrm{CH}_{4}$. Central to the experiments was the interaction and mixing between $\mathrm{CO}_{2}$ in its 
supercritical state and the $\mathrm{CH}_{4}$ in the reservoir given that the $\mathrm{CO}_{2}$ plume occupies the lower echelon of the $\mathrm{CH}_{4}$ zone due to its higher density at supercritical condition as we have iterated in our previous works. Vertical and horizontal flows of the $\mathrm{CO}_{2}$ plume are the two extreme flow conditions in terms of propagation direction.

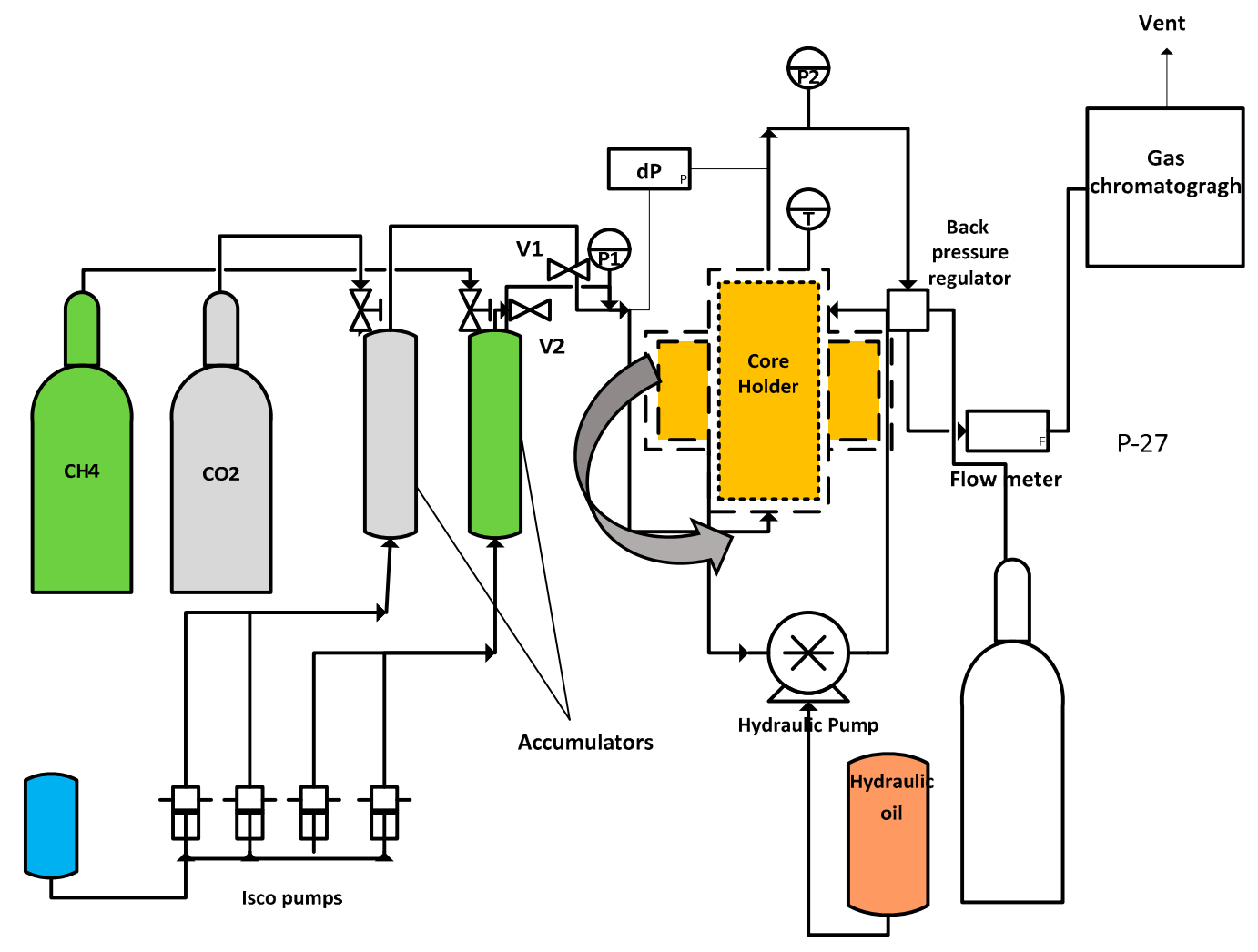

Figure 2. Core flooding set up schematics.

(3) Data analysis

A single parameter diffusion equation was used by [11] in the description of the longitudinal dispersion coefficient for gas flow in porous media which is shown in Equation (1):

$$
K_{l} \frac{\partial^{2} C}{\partial x^{2}}-u \frac{\partial C}{\partial x}=\frac{\partial C}{\partial t},
$$

where $C-\mathrm{CO}_{2}$ concentration at time $t$, location $x, K_{l}$-the longitudinal dispersion coefficient, and $u$ is the interstitial velocity. The dimensionless for of Equation (1) is written as

$$
\frac{1}{P_{e}} \frac{\partial^{2} C}{\partial x_{D}^{2}}-\frac{\partial C}{\partial x_{D}}=\frac{\partial C}{\partial t_{D}}
$$

where each parameter is defined in Table 3.

Table 3. Dimensionless parameters.

\begin{tabular}{cc}
\hline Parameter & Expression \\
\hline$P_{e}$ & $\frac{u L}{K_{l}}$ \\
$t_{D}$ & $\frac{t u}{L}$ \\
$x_{D}$ & $\frac{x}{L}$ \\
$u$ & $\frac{Q}{\pi r^{2} \phi}$ \\
\hline
\end{tabular}


This one-dimensional advection-dispersion (ADE) equation was used to measure/evaluate, analytically, the longitudinal dispersion of $\mathrm{CO}_{2}$ into $\mathrm{CH}_{4}$ using the solution of the equation and also assuming that the dispersion coefficient and interstitial velocity of the displacing species are not affected by the concentration. With the following boundary conditions, the initial condition $-C=0$ at $t_{D}=0$, boundary conditions- $-C=1$ at $x_{D}=0, C \rightarrow 0$ as $x_{D} \rightarrow \infty$, the dimensionless solution to (Equation (1)) thus becomes [12-14]

$$
C=\frac{1}{2}\left\{\operatorname{erfc}\left(\frac{x_{D}-t_{D}}{2 \sqrt{t_{D} / P_{e}}}\right)+e^{P_{e} x_{D}} \operatorname{erfc}\left(\frac{x_{D}+t_{D}}{2 \sqrt{t_{D} / P_{e}}}\right)\right\}
$$

Using the obtained concentration profiles from core flooding experiments, Equation (3) was used to fit the results from the experiments and the dispersion coefficient was obtained analytically using the least squares regression method with the dispersion coefficient as the fitting parameter.

Perkins and Johnston (1963) described the medium Péclet number denoted by $P_{e x}$, which defines the displacement mechanism that is dominant in gas dispersion in porous media as

$$
P_{e x}=\frac{u_{m} d}{D}
$$

where $D$-diffusion coefficient $\left(\mathrm{m}^{2} / \mathrm{s}\right), P_{e m}$-medium Péclet number, $u_{m}$-mean interstitial velocity $(\mathrm{m} / \mathrm{s})$, and $d$ is the characteristic length scale of mixing in the porous medium. Largely, at the values of $P_{e m}<0.1$, diffusion mixing dominates and, equally, at $P_{e x}>10$, advective mixing dominates during the gas dispersion process. In this range of $P_{e x},[15]$ related diffusion to dispersion coefficients as presented in (Equation (6)):

$$
\frac{K_{l}}{D}=\frac{1}{\tau}+\alpha \frac{u_{m}^{n}}{D}
$$

where $\alpha$ represents the dispersivity of the porous medium in $m, \tau$ is the tortuosity of the porous medium, and $n$ is an exponent.

Additionally, [16-18] reported a correlation between the molecular diffusion coefficient, pressure, and temperature to obtain accurate diffusivity at conditions relevant to EGR as follows:

$$
D=\frac{\left(-4.3844 \times 10^{-13} p+8.55440 \times 10^{-11}\right) T^{1.75}}{p},
$$

where $D\left(\mathrm{~m}^{2} / \mathrm{s}\right)$ is the molecular diffusion coefficient of $\mathrm{CO}_{2}$ in $\mathrm{CH}_{4}$ pressure $p(\mathrm{MPa})$ and at temperature $T(\mathrm{~K})$.

\section{Results and Discussion}

The core flooding experiments were carried out at $1300 \mathrm{psig}$, and $50{ }^{\circ} \mathrm{C}$ in both vertical and horizontal orientations for each core sample. The interstitial velocities were varied according to individual core samples given their different porosities. A range of interstitial velocities was based on the injection rates in our previous work [8], which were evaluated using Equation (7):

$$
u=\frac{Q}{\phi A}
$$

where $Q$-injection rate in $\mathrm{m}^{3} / \mathrm{s}, A$-cross-sectional area of core sample, and $\varphi$-porosity of core sample. The results are shown in Table 4. 
Table 4. Interstitial velocities employed in each core sample.

\begin{tabular}{cccc}
\hline SN & Core Sample & Porosity (\%) & Interstitial Velocity $(\mu \mathrm{m} / \mathbf{s})$ \\
\hline 1 & Grey Berea & 20.10 & $33.5-83.8$ \\
2 & Bandera Grey & 17.20 & $39.3-98.3$ \\
3 & Buff Berea & 26.27 & $26.2-65.6$ \\
\hline
\end{tabular}

Accordingly, the results of each individual core sample will be presented, discussed, and analysed based on the injection orientation and the interstitial velocity. Grey Berea core sample will be presented first, followed by Bandera Grey and, finally, Buff Berea.

The mole fractions of the injected $\mathrm{CO}_{2}$ were used to develop the concentration profile which assesses rate of mixing between the injected $\mathrm{CO}_{2}$ and the $\mathrm{CH}_{4}$, in situ, using Equation (3) to fit the equation to the experimental data and varying $K_{L}$, which is the longitudinal dispersion coefficient (keeping the interstitial velocity constant as assumed in the 1D ADE), until the analytical solution fits the experimental results. The $L_{\exp }$ was also adjusted in the regression to provide a good fit as carried out by [16] and adopted by [17]. Least square regression analysis was the method used in the curve fitting process.

Curve fitting was carried out using OriginPro 8 software and the curve-fitted concentration profiles for each $u$ for Grey Berea core sample are shown in Figures 3 and 4 for horizontal and vertical orientation respectively. The dispersion coefficients were evaluated, and it was shown that the $K_{L}$ increases with increase in the interstitial velocity. This was done for all core samples and injection orientation experiments in this investigation. There was early breakthrough at higher values of $u$ run and a late breakthrough at lower values as expected in all the core samples. The fitting of the 1D ADE to the experimental results was meagre as a result of systemics like entry and exit effects as described in the works of [17]. This was noted for all the runs in the entire experiments. However, these do not affect the evaluation of the parameter i.e., dispersion coefficient. For all subsequent experiments, these systemic effects were noticed and are presented as such.

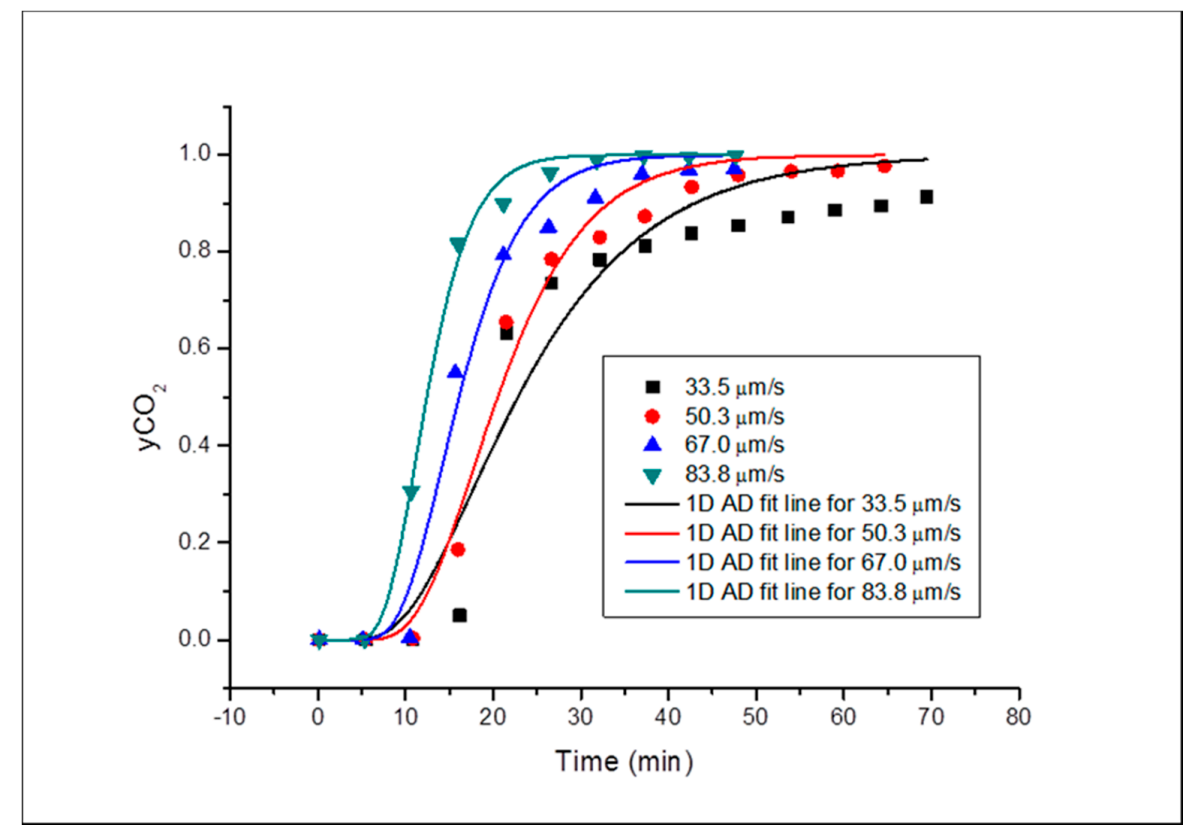

Figure 3. Concentration profile for Grey Berea in horizontal orientation.

Using Equation (6), the diffusion coefficients, $D$, were evaluated at the experimental conditions. This is essential when describing the dispersivity, $\alpha$, and the $P_{e x}$ of the core sample, and also when comparing the results to those in literature, which will further reaffirm the accuracy of the experiments. 
The dispersivity can be analytically evaluated by fitting Equation (5) to the plot of $u / D$ against $k / D$ which is a straight line as shown in Figure 4.

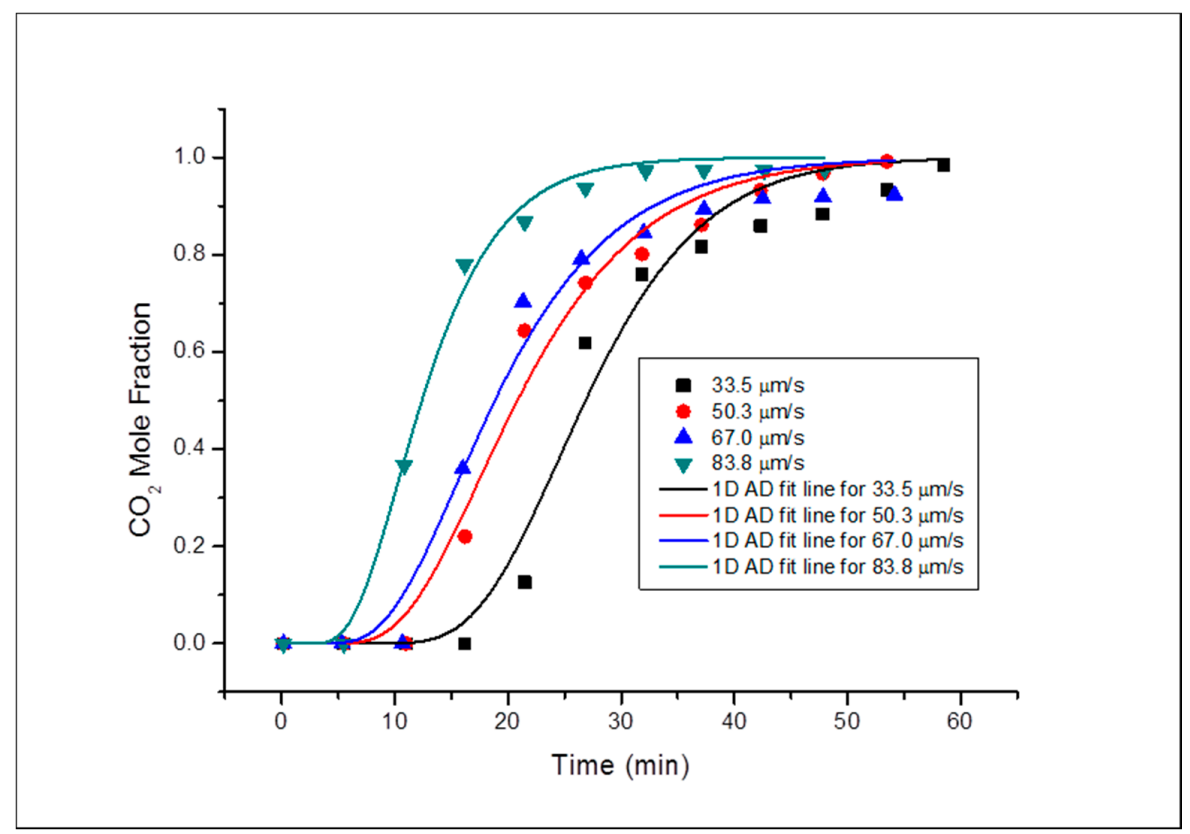

Figure 4. Concentration profile for all Grey Berea runs vertical orientation.

The fitted concentration profiles of the subsequent core sample (Buff Bera) is shown in Figures 5 and 6, for horizontal and vertical orientation respectively, which also showed meagre fitting as a result of systemic errors as seen in Grey Berea.

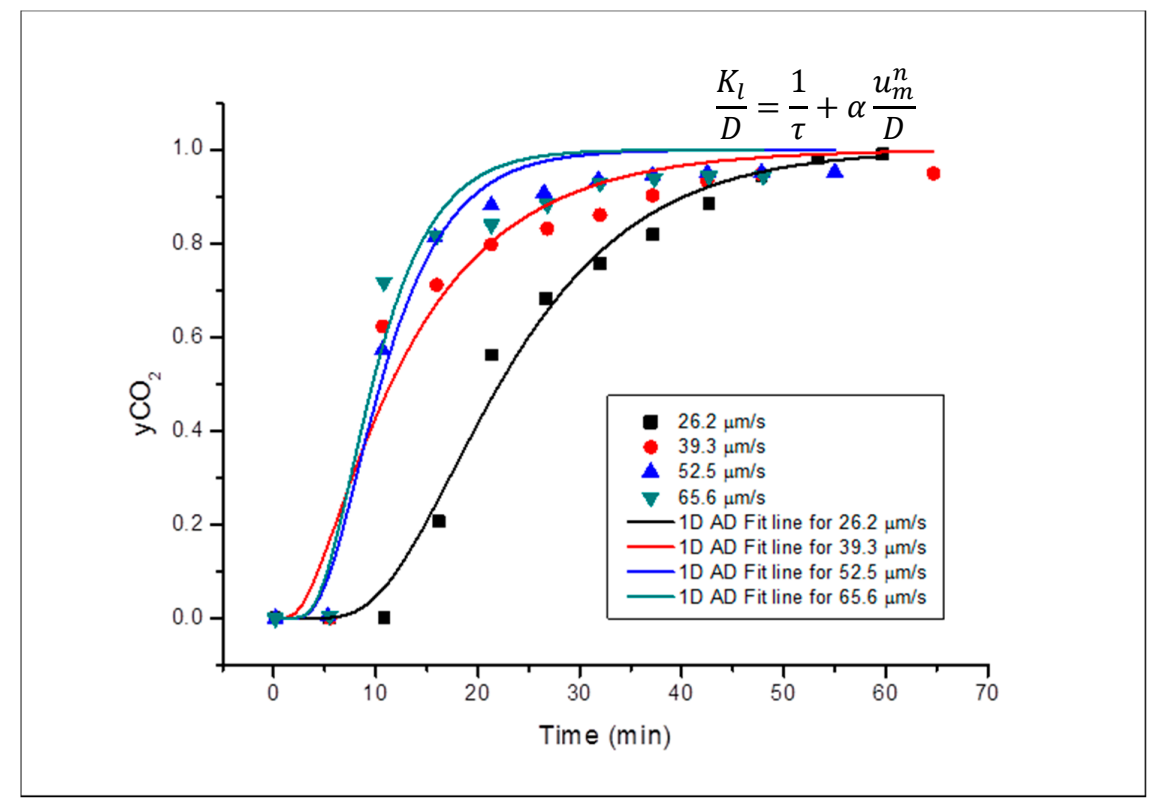

Figure 5. Concentration profile of all the runs for Buff Berea in horizontal orientation. 


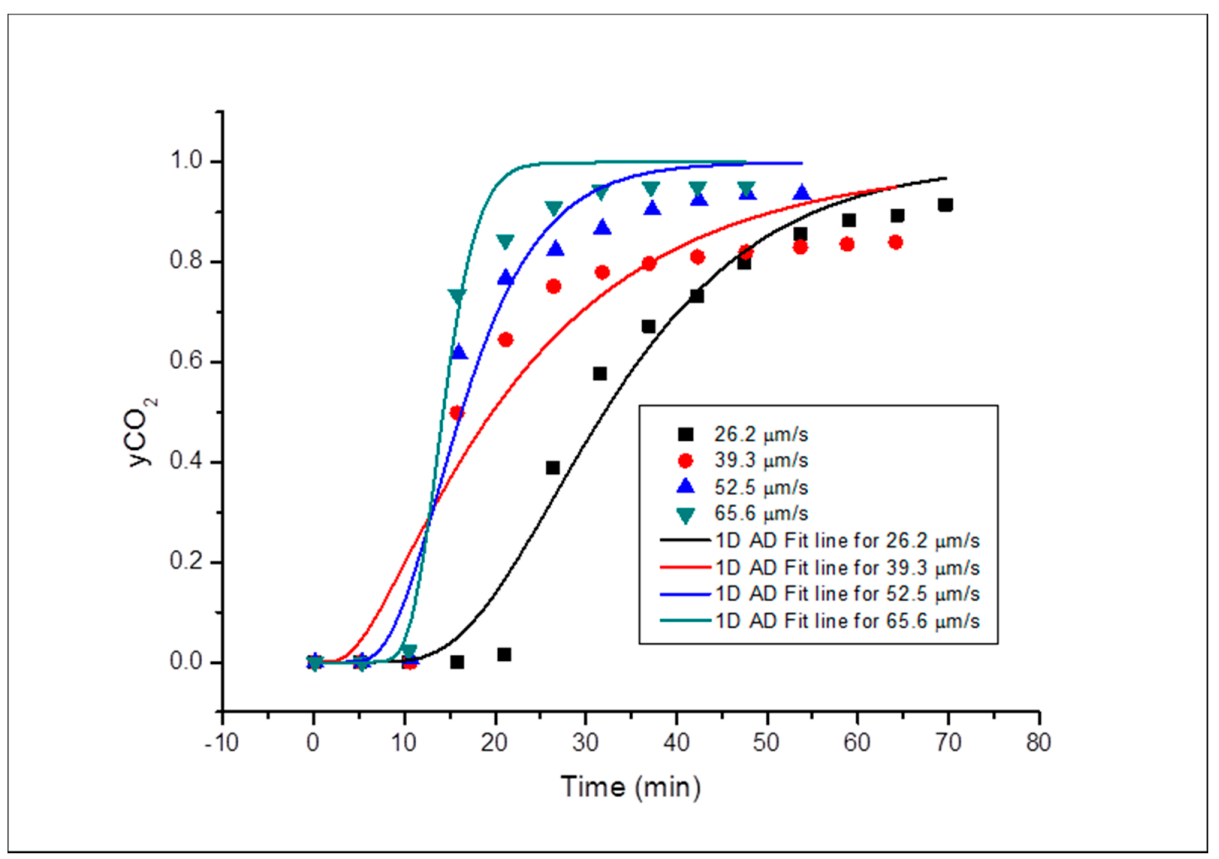

Figure 6. Concentration profile for all Buff Berea runs in vertical orientation.

Similarly, Bandera Grey core sample fitted horizontal and vertical orientation concentration profiles are shown in Figures 7 and 8. The profiles are steeper than the previous ones obtained for the core samples (Buff Berea and Grey Berea) because of the instant mixing during the displacement process due to higher interstitial velocities.

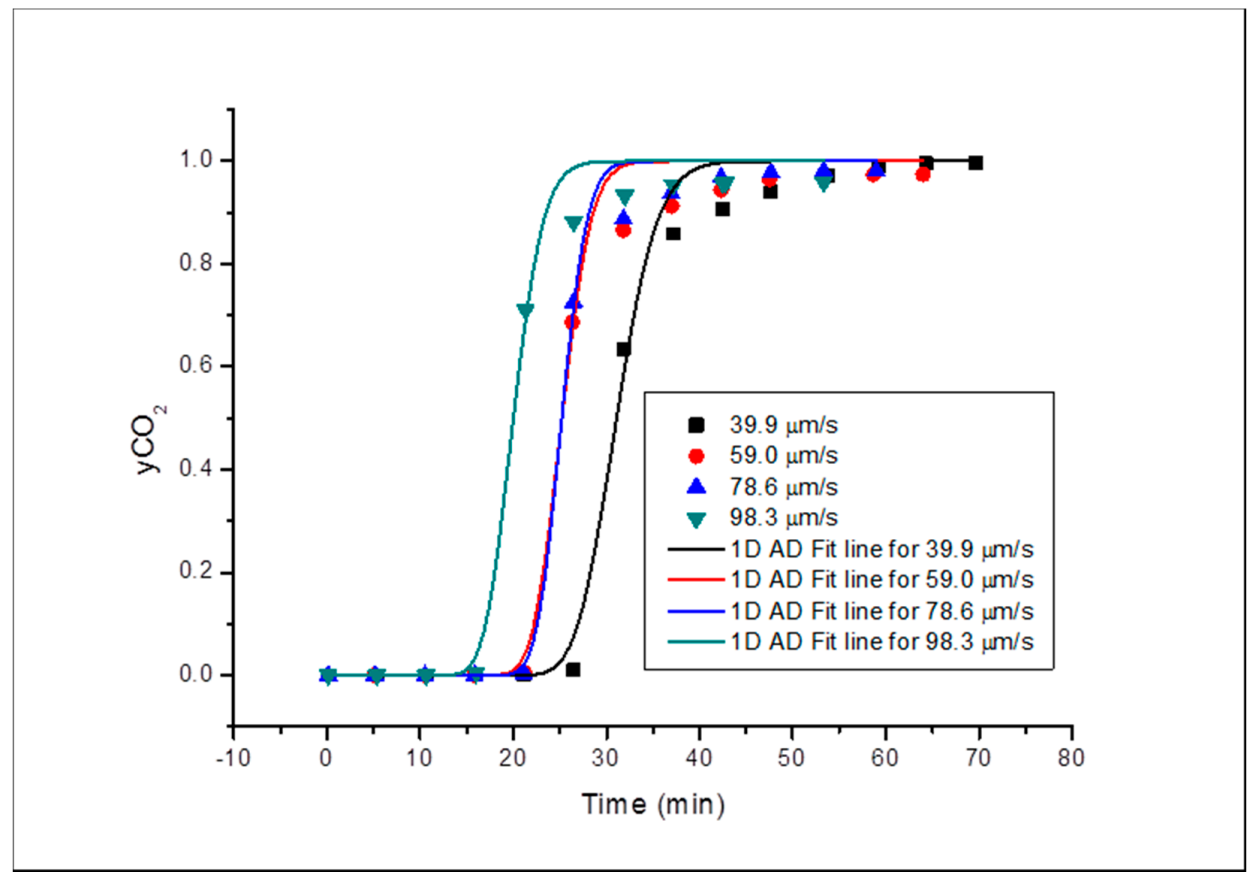

Figure 7. Concentration profile for Bandera Grey in horizontal orientation. 


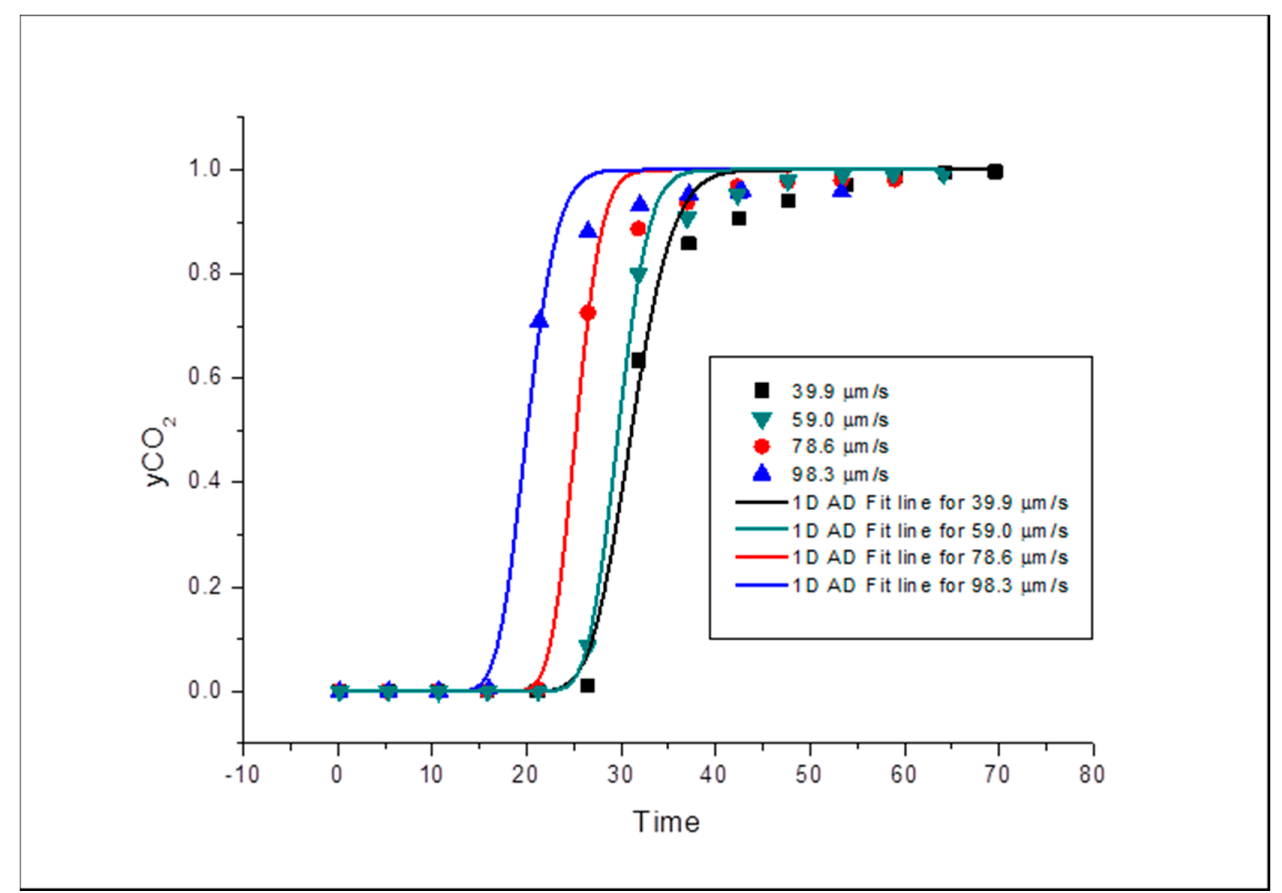

Figure 8. Concentration profile for Bandera Grey runs in vertical orientation.

The works of $[16,18,19]$ presented values of the apparent dispersivity in consolidated porous media that were generally smaller than $0.01 \mathrm{ft}(0.003 \mathrm{~m})$. Hughes et al. (2012) obtained dispersivity in a range of 0.0001 to $0.0011 \mathrm{~m}$ using a core sample (Donny brook) with similar petrophysical properties as the ones used in this work. This provided a practical input variable for EGR simulations. As dispersivity is a very important porous media property, its accurate determination can provide a befitting technique to establish the optimum injection rate of the $\mathrm{CO}_{2}$ for a better simulation of the fluid flow in the matrix of the reservoir during EGR. All the dispersivity obtained (from each experiment carried out in this study are well within those obtained from literature. This was evaluated from Equation (5) as earlier stated-a straight-line equation. This further demonstrates the reliability of the experiments.

The tortuosity (inverse of the intercept on the y-axis of the straight line) was similar in both cases (Figures 9-11), for Grey Berea, indicating that regardless of the injection orientation of the core sample, the tortuosity (a property of the core sample) remained unchanged. This shows that core orientation does not alter the pathways of the matrix of the porous media when fluids traverse through the porous medium, further attributing the fluid behaviour to mainly a function of the fluid properties and not the porous media. This was also true for Buff Berea depicted in Figures 12 and 13, and a comparison between the vertical and horizontal dispersivity is shown in Figure 14. 


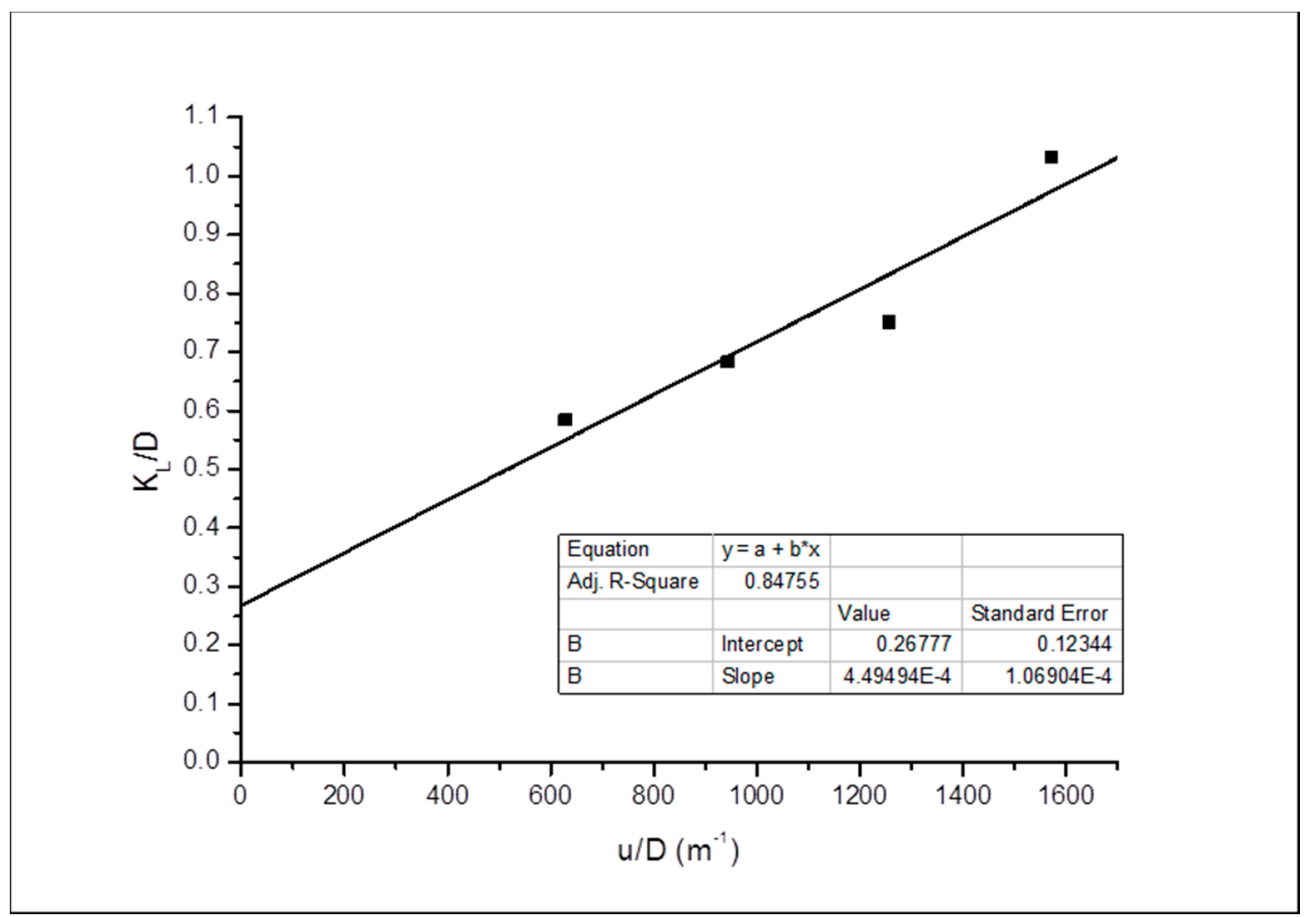

Figure 9. Dispersion to diffusion coefficient ratio against interstitial velocity for Grey Berea (vertical).

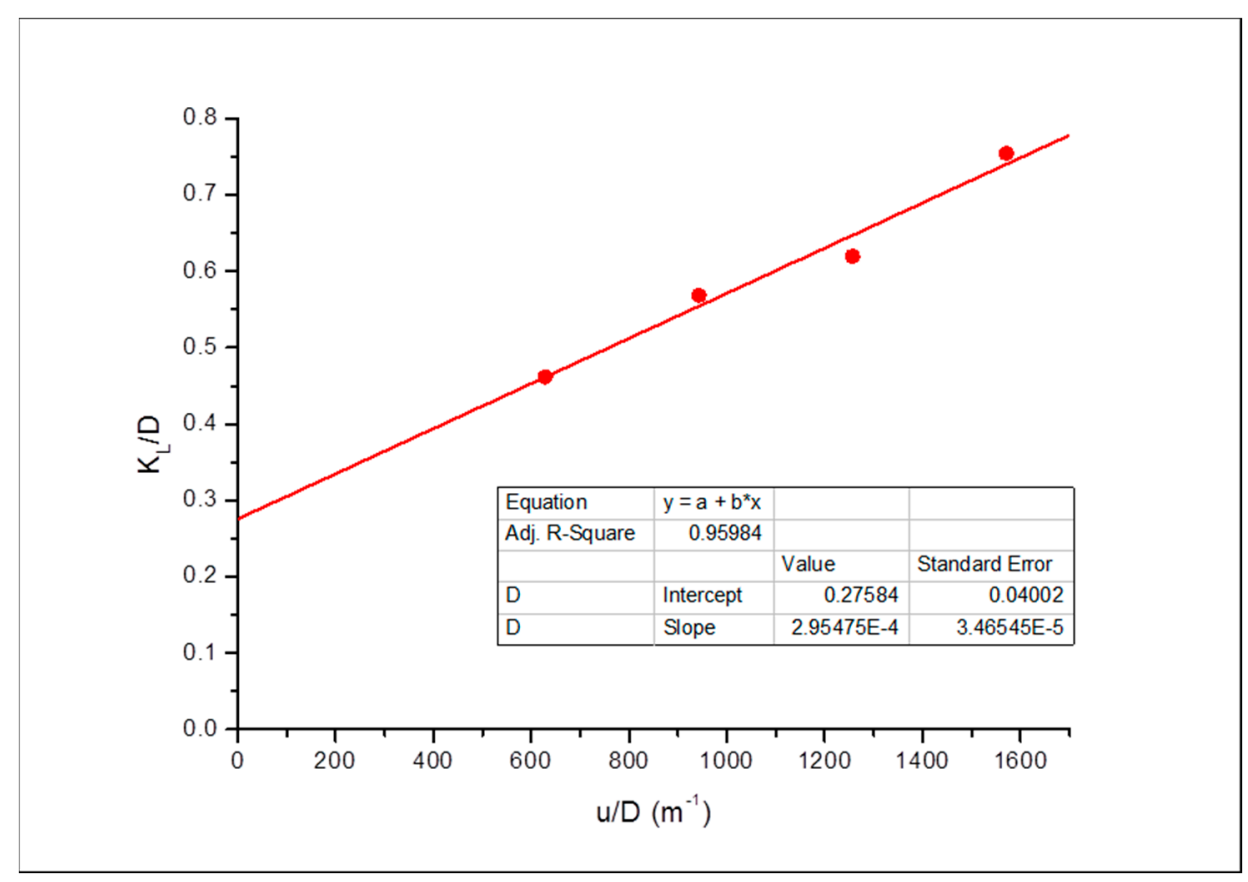

Figure 10. Dispersivity evaluation using the dispersion coefficient and interstitial velocity for Grey Berea (horizontal). 


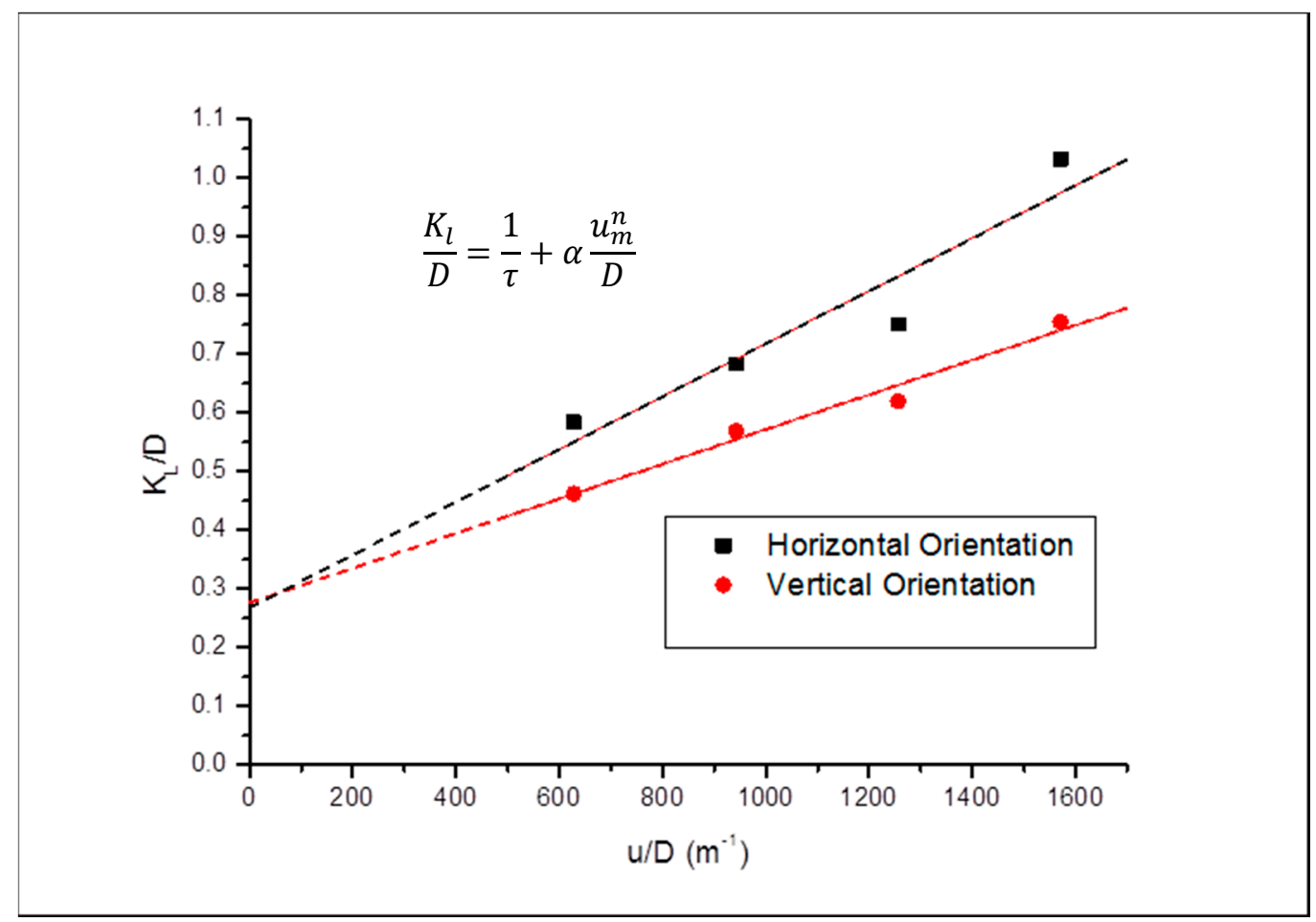

Figure 11. Comparison of the dispersivities in both orientations for Grey Berea.

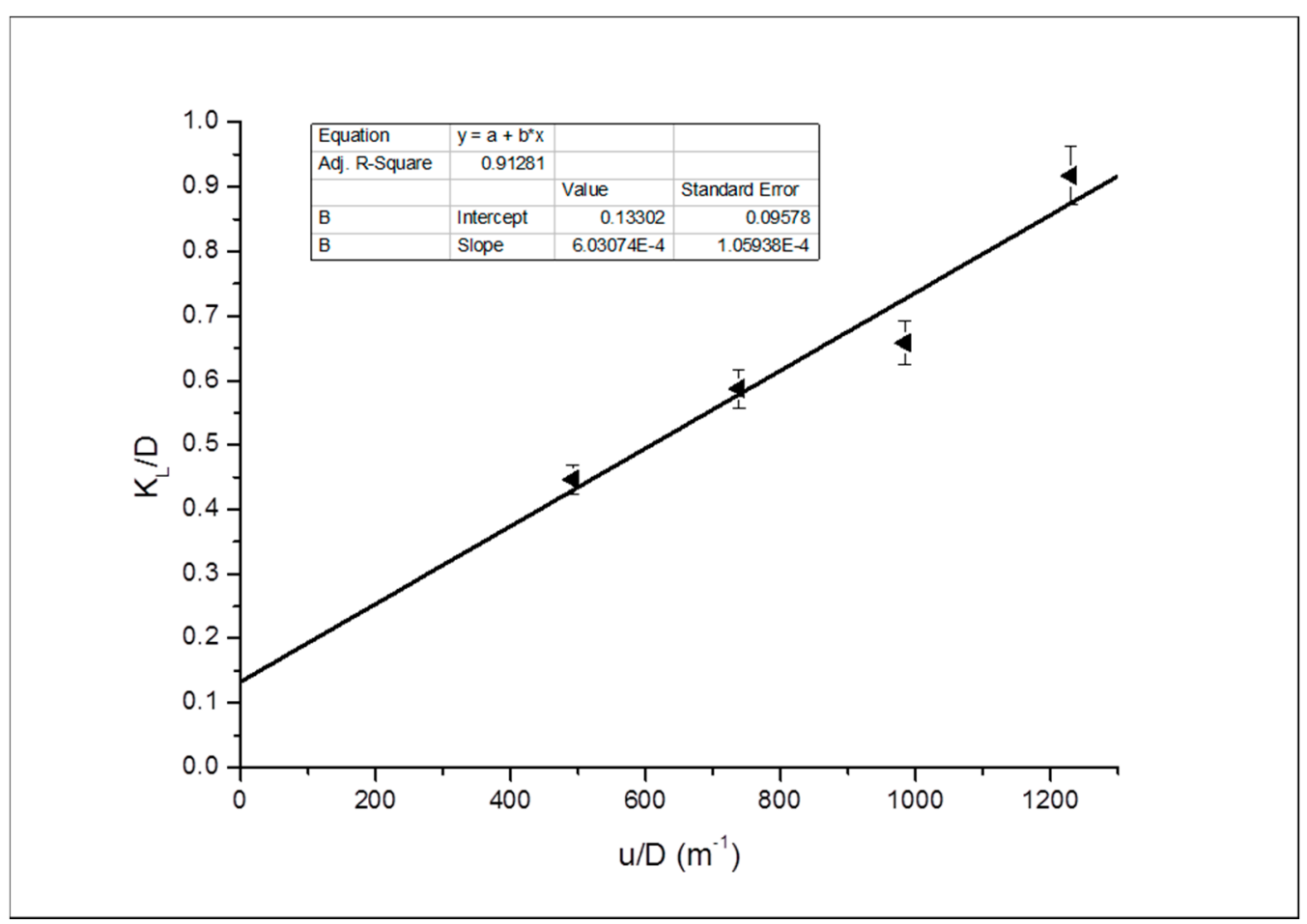

Figure 12. Dispersivity evaluation for Buff Berea in the horizontal orientation. 


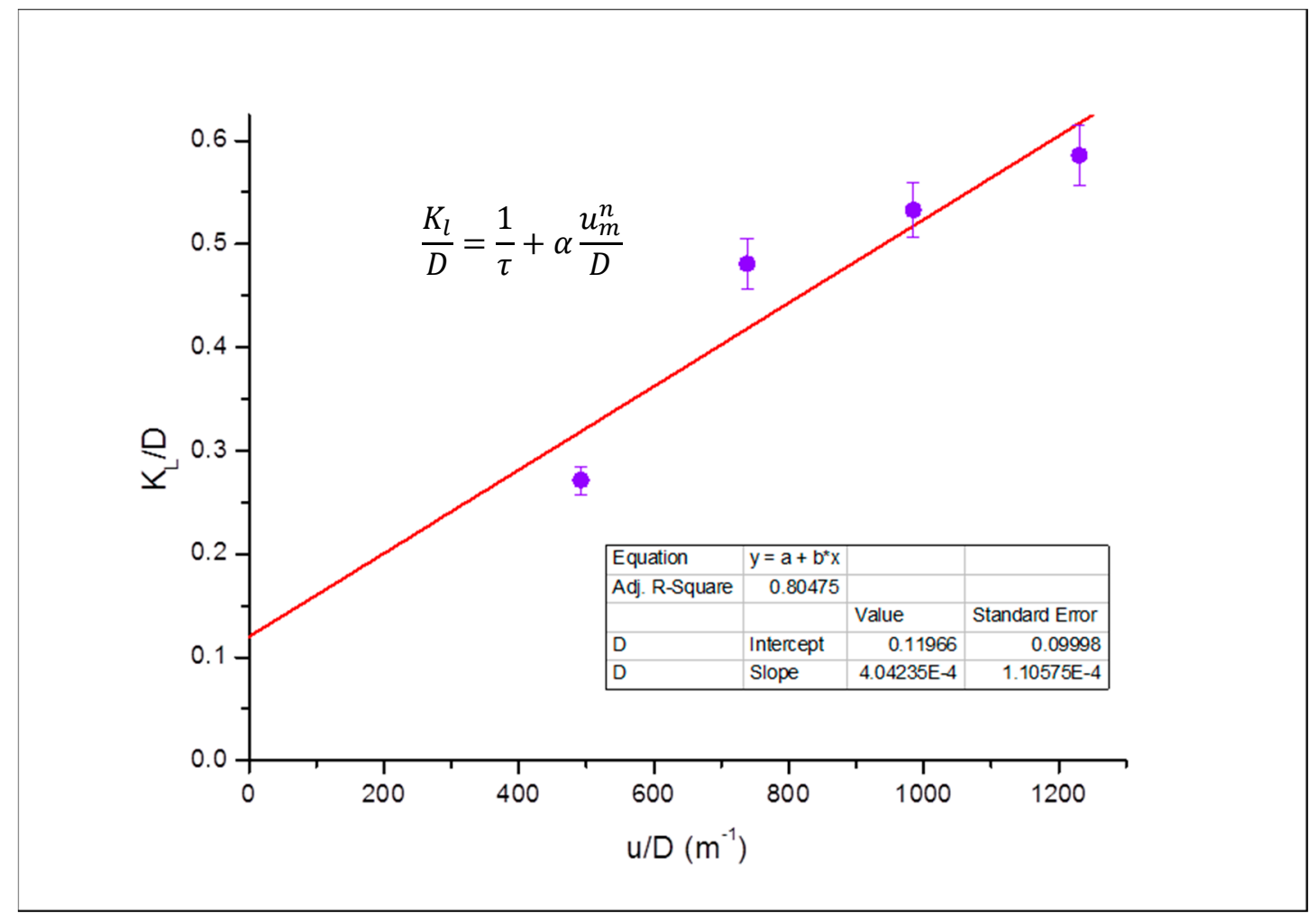

Figure 13. Dispersivity evaluation for Buff Berea in the vertical orientation.

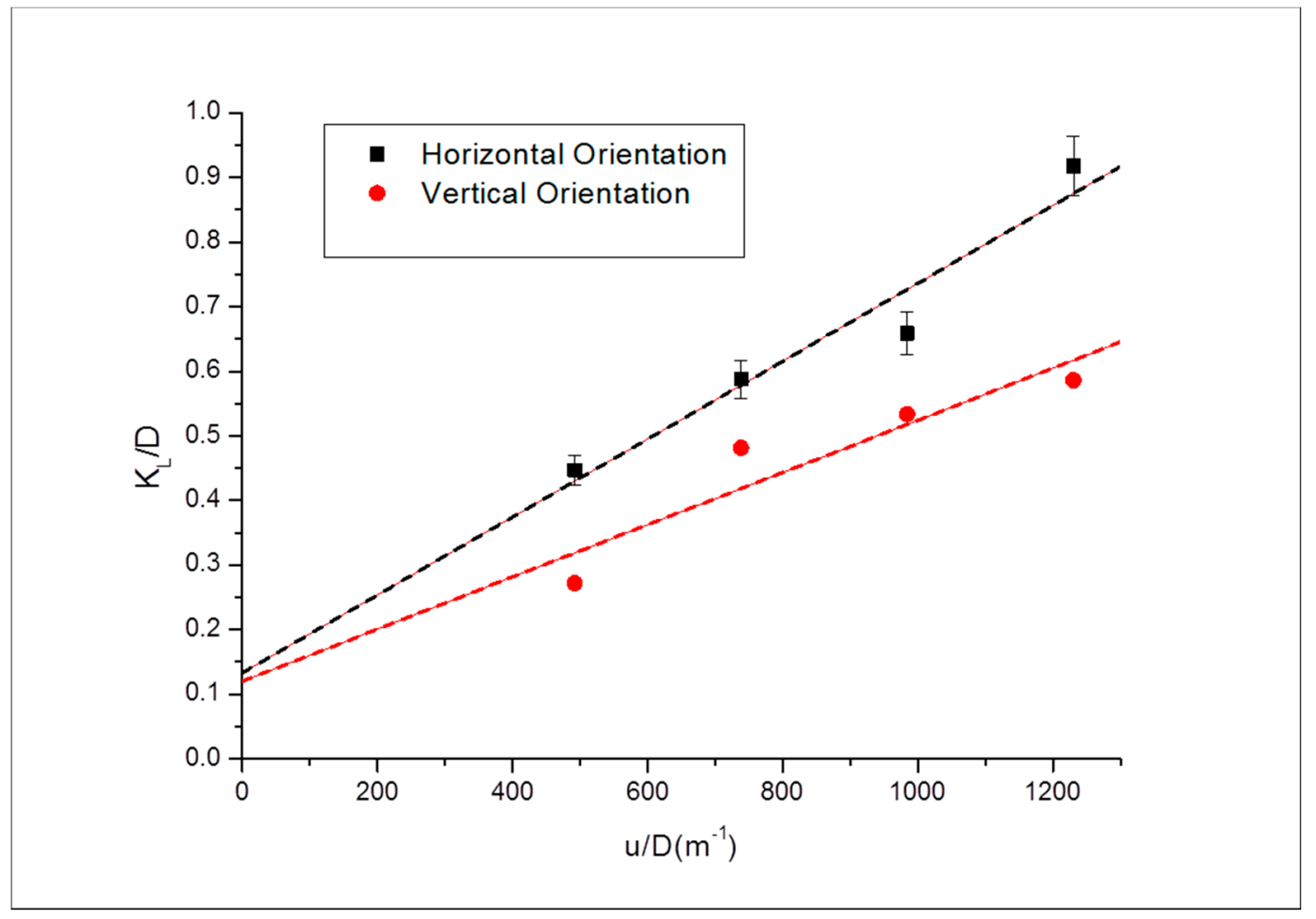

Figure 14. Comparison of the dispersivities in both orientations for Buff Berea.

Bandera Grey core sample exhibited similar trend as the previous core samples discussed in terms of dispersivity as shown in Figures 15-17. Dispersivity, however, is highest in Bandera Grey compared to the other core samples. This can be attributed to the grain arrangement of the core sample and the structure of the pore matrix which is tightly packed with its characteristic low permeability. 


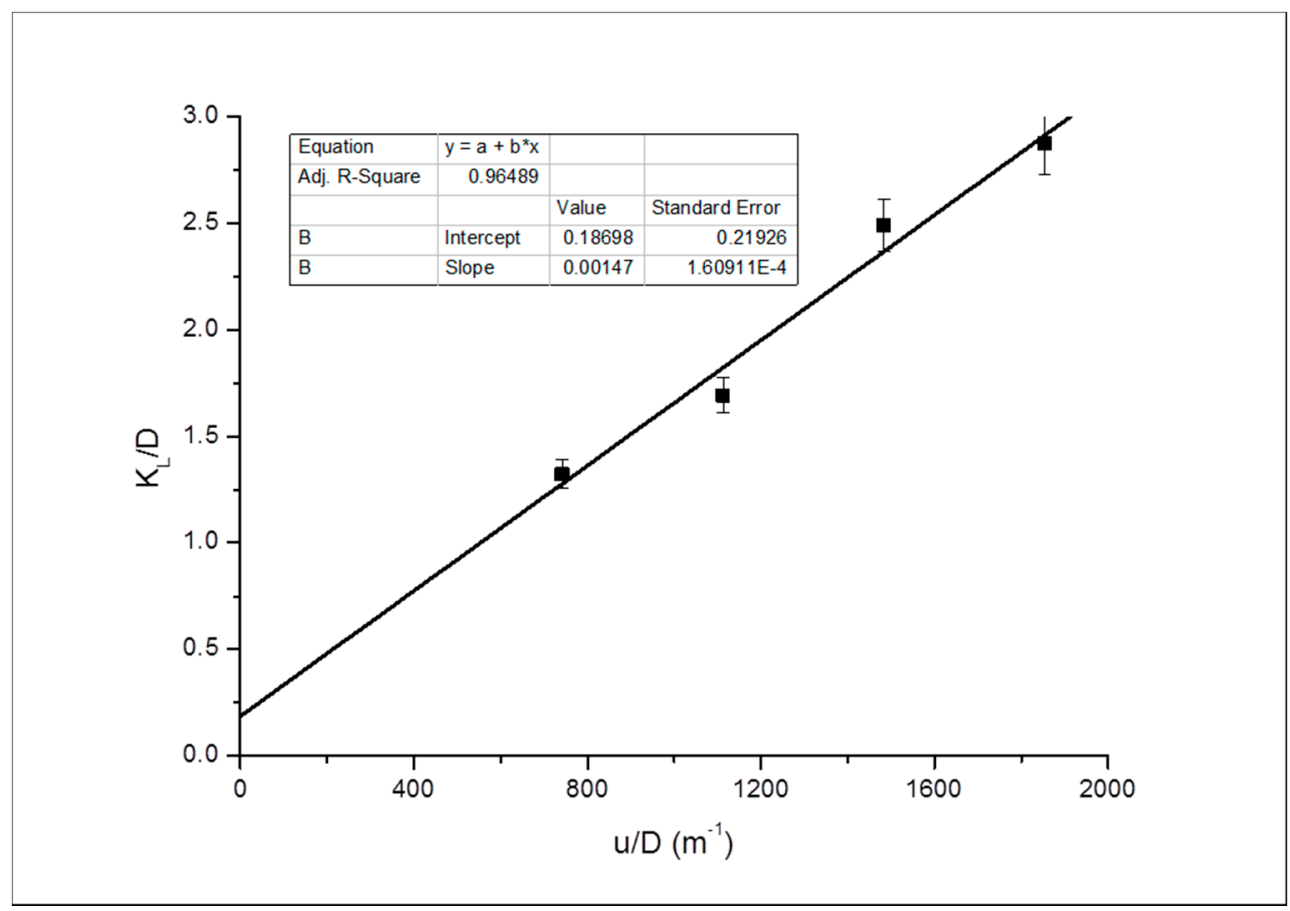

Figure 15. Dispersivity evaluation for Bandera Grey in the horizontal orientation.

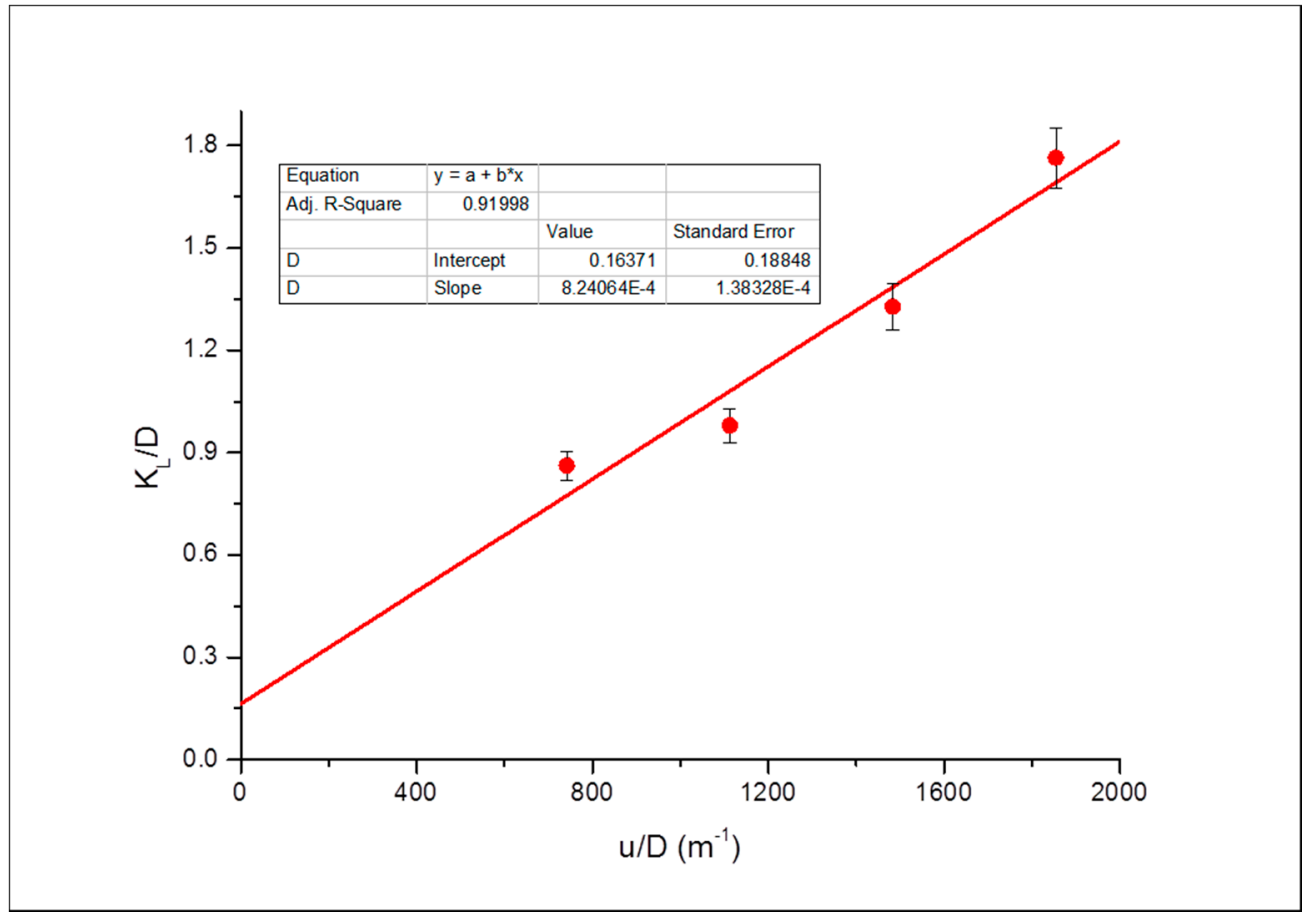

Figure 16. Dispersivity evaluation for Bandera Grey in the vertical orientation. 


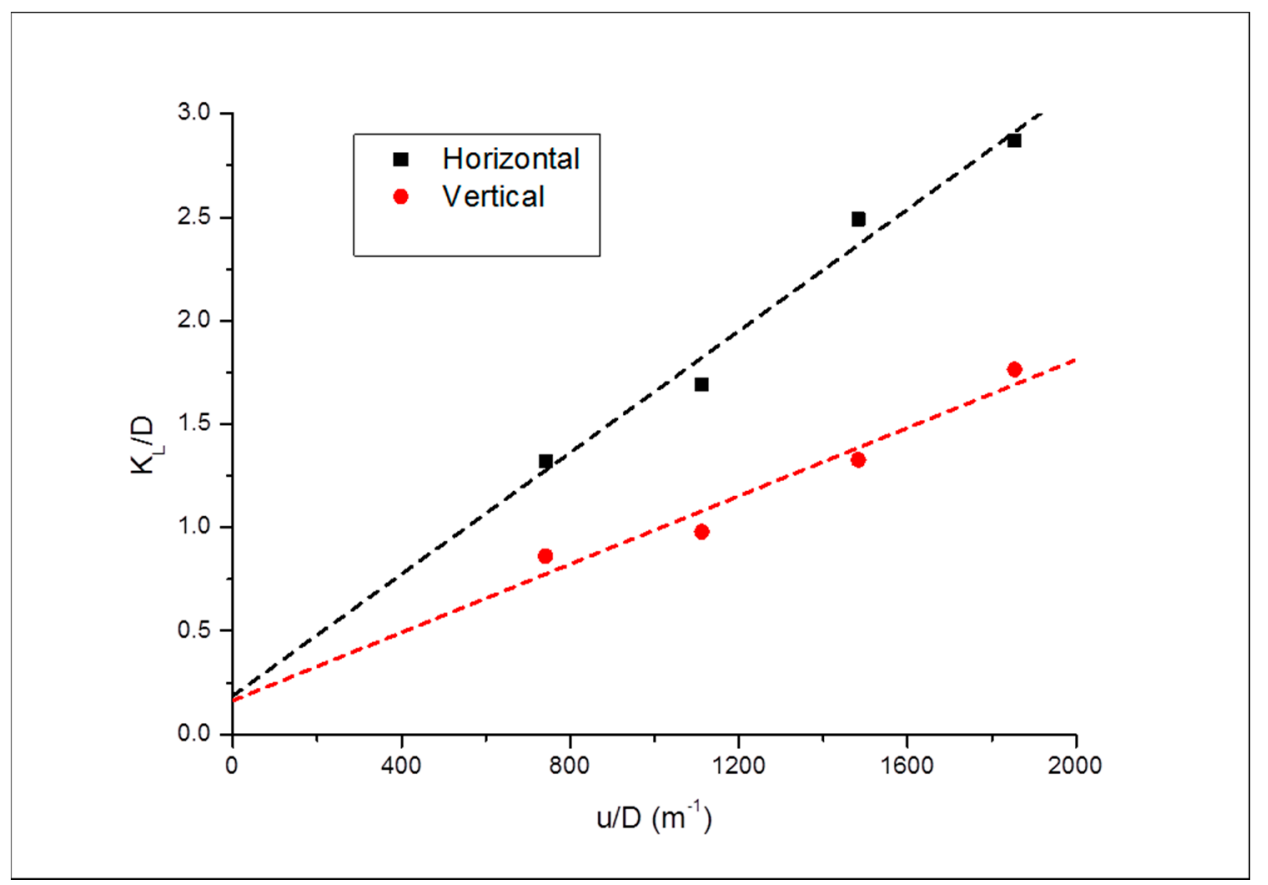

Figure 17. Comparison of the dispersivities in orientations for Bandera Grey.

There certainly are similarities between the vertical and horizontal orientations in all the core samples used in this study in terms of dispersion coefficient. This discussion is highlighted in the next section, which summarises the dispersion coefficient variation with the permeability.

\section{Summary of the Dispersion Coefficient Investigation}

A summary of the dispersion coefficients for all the core samples is shown in Table 5.

Table 5. Summary of all dispersion coefficients.

\begin{tabular}{cccc}
\hline \multirow{2}{*}{ Core Sample } & $\mathcal{U}(\boldsymbol{\mu} \mathbf{m} / \mathbf{s})$ & \multicolumn{2}{c}{$\left.\boldsymbol{K}_{\mathbf{L}} \mathbf{( 1 0}^{-\mathbf{8}} \mathbf{~ m}^{\mathbf{2}} \mathbf{s}\right)$} \\
\cline { 3 - 4 } & & Horizontal & Vertical \\
\hline \multirow{3}{*}{ Bandera $(k=16.08 \mathrm{md})$} & 39.3 & 7.01 & 4.56 \\
& 59.0 & 8.97 & 5.19 \\
& 78.6 & 13.20 & 7.03 \\
& 98.3 & 15.23 & 9.35 \\
\hline \multirow{3}{*}{ Grey Berea $(k=217.04 \mathrm{md})$} & 33.5 & 3.11 & 2.46 \\
& 50.3 & 3.64 & 3.03 \\
& 67.0 & 4.01 & 3.70 \\
& 83.8 & 5.51 & 4.02 \\
\hline \multirow{3}{*}{ Buff Berea $(k=560.63 \mathrm{md})$} & 26.2 & 2.38 & 1.44 \\
& 39.3 & 3.13 & 2.56 \\
& 52.5 & 3.51 & 2.84 \\
& 65.6 & 4.89 & 3.12 \\
\hline
\end{tabular}

Dispersion coefficient generally decreases with increase in permeability as seen in Table 5. Hence, the core sample with the least permeability (Bandera Grey) showed a significantly higher dispersion coefficient. Another realisation from the Table is that in all the runs, those in the horizontal orientation appear to have the higher dispersion coefficient compared to the vertical counterparts. This can be attributed to the effect of gravity on the $\mathrm{CO}_{2}$ as it traverses the core sample. Also, since the interstitial velocity is a function of porosity, the core sample with the most porosity had the lowest interstitial velocity and, hence, a lower dispersion coefficient at lower injection rates. The dispersion 
coefficient increases significantly at higher injection rates in all the runs regardless of the orientation. Furthermore, in the higher permeability core sample, the interaction between the injected $\mathrm{CO}_{2}$ and the nascent $\mathrm{CH}_{4}$ was limited as the $\mathrm{CO}_{2}$ interstitial velocity was lower and, thus, the dispersion coefficient was lower compared to the subsequent core samples. The opposite was realised in the low permeability sample with the highest interstitial velocity was characterised by higher agitation of the gas molecules within the porous medium which eventually led to higher interaction and mixing and of course higher dispersion coefficient-the rate of mixing. Permeability is one of the vast factors that influence dispersion.

The dispersivity also increases with increase in permeability. This being a function of the core sample is evident with this trend, as the absolute permeability of the core sample is also a property of the core sample. Basically, the higher the permeability of the core sample, the higher the rate of mixing when $\mathrm{CO}_{2}$ is injected to displace $\mathrm{CH}_{4}$. However, dispersivity is scale-dependent $[20,21]$ and, albeit being at laboratory scale, this finding is an indication of the effects of the petrophysical properties on the mixing taking place during EGR. Finding the right injection scenario is vital in achieving the best recovery efficiency whilst storing substantial volumes of $\mathrm{CO}_{2}$.

\section{Conclusions}

The effects of permeability on the dispersion coefficient have been shown to be significant during the displacement of $\mathrm{CH}_{4}$ by supercritical $\mathrm{CO}_{2}$. This effect was more pronounced in the horizontal orientation compared to the vertical orientation which was attributed to the gravity effects on the supercritical $\mathrm{CO}_{2}$ as it traverses the core sample. The magnitude difference in the dispersion coefficients was about $20 \%-30 \%$ higher in the horizontal orientation compared to the vertical orientation. Bandera Grey, with the lowest permeability and porosity, exhibited a wider contrast $(40 \%-50 \%)$ between the dispersion coefficients in both orientations as a result of higher interstitial velocity and tortuosity of the core sample compared to the other core samples tested. Therefore, the effect of permeability and orientation on mixing between the displacing and displaced gas during EGR are vital. This study highlights the influence of the permeability variation and plume propagation orientation on the mixing between $\mathrm{CO}_{2}$ and $\mathrm{CH}_{4}$ during EGR. It provides reservoir engineers with an insight into characterising gas systems during the design of the EGR technology. The injection rates, injection pressures, and dip angle of injection from the injector to the producer can be evaluated accurately during simulation studies prior to field scale application and implementation. Inclusion of such hysteresis will provide a better representation of the injection process given that the main reason why EGR has not been widely adopted is the its economic drawback brought on by incessant mixing and contamination of the recovered $\mathrm{CH}_{4}$.

Author Contributions: Conceptualization, M.K.A. and A.J.A.; Methodology, M.K.A.; Data software tools analysis, A.A.-O.; Investigation, M.K.A.; writing—original draft preparation, M.K.A.; writing—review and editing, M.B.; visualization, A.A.-O.; supervision, G.G.N.

Funding: This research received no external funding.

Acknowledgments: The authors would like to show gratitude to Petroleum Technology Development Fund Nigeria (PTDF) for the studentship.

Conflicts of Interest: The authors declare no conflict of interest.

\section{References}

1. Ziyarati, M.T.; Bahramifar, N.; Baghmisheh, G.; Younesi, H.; Younessi, H. Greenhouse gas emission estimation of flaring in a gas processing plant: Technique development. Process. Saf. Environ. Prot. 2019, 123, 289-298. [CrossRef]

2. Abas, N.; Kalair, A.; Khan, N. Review of fossil fuels and future energy technologies. Futures 2015, 69, 31-49. [CrossRef]

3. Wegener, M.; Amin, G.R. Minimizing Greenhouse Gas Emissions using Inverse DEA with an Application in Oil and Gas. Expert Syst. Appl. 2018, 122, 369-375. [CrossRef] 
4. Vilcáez, J. Numerical modeling and simulation of microbial methanogenesis in geological $\mathrm{CO}_{2}$ storage sites. J. Pet. Sci. Eng. 2015, 135, 583-595. [CrossRef]

5. Kalra, S.; Wu, X. $\mathrm{CO}_{2}$ injection for Enhanced Gas Recovery. In Proceedings of the SPE Western North American and Rocky Mountain Joint Meeting, Denver, CO, USA, 17-18 April 2014; pp. 16-18.

6. Abba, M.K.; Abbas, A.J.; Nasr, G.G. Enhanced Gas Recovery by $\mathrm{CO}_{2}$ Injection and Sequestration: Effect of Connate Water Salinity on Displacement Efficiency. In Proceedings of the SPE Abu Dhabi International Petroleum Exhibition \& Conference, Abu Dhabi, UAE, 13-16 November 2017.

7. Abba, M.K.; Al-Othaibi, A.; Abbas, A.J.; Nasr, G.G. Effects of gravity on flow behaviour of supercritical $\mathrm{CO} 2$ during enhanced gas recovery and sequestration. In Proceedings of the Fifth $\mathrm{CO} 2$ Geological Storage Workshop, Utrecht, The Netherlands, 21-23 November 2018.

8. Abba, M.K.; Al-Othaibi, A.; Abbas, A.J.; Nasr, G.G.; Mukhtar, A. Experimental investigation on the impact of connate water salinity on dispersion coefficient in consolidated rocks cores during Enhanced Gas Recovery by $\mathrm{CO}_{2}$ injection. J. Nat. Gas Sci. Eng. 2018, 60, 190-201. [CrossRef]

9. Abba, M.K.; Abbas, A.J.; Al-Othaibi, A.; Nasr, G.G. Enhanced Gas Recovery by $\mathrm{CO}_{2}$ Injection and Sequestration: Effects of Temperature, Vertical and Horizontal Orientations on Dispersion Coefficient. In Proceedings of the Abu Dhabi International Petroleum Exhibition \& Conference, Abu Dhabi, UAE, 12-15 November 2018.

10. Liu, S.; Song, Y.; Zhao, C.; Zhang, Y.; Lv, P.; Jiang, L.; Liu, Y.; Zhao, Y. The horizontal dispersion properties of $\mathrm{CO}_{2}$-CH4in sand packs with CO2displacing the simulated natural gas. J. Nat. Gas Sci. Eng. 2018, 50, $293-300$. [CrossRef]

11. Newberg, M.; Foh, S. Measurement of Longitudinal Dispersion Coefficients for Gas Flowing Through Porous Media. In Proceedings of the SPE Gas Technology Symposium, Dallas, TX, USA, 13-15 June 1988; pp. 5-9.

12. Mamora, D.D.; Seo, J.G. Enhanced Recovery by Carbon Dioxide Sequestration in Depleted Gas Reservoirs. In Proceedings of the SPE Annual Technical Conference and Exhibition, San Antonio, TX, USA, 29 September-2 October 2002; pp. 1-9.

13. Nogueira, M.; Mamora, D.D. Effect of Flue-Gas Impurities on the Process of Injection and Storage of $\mathrm{CO}_{2}$ in Depleted Gas Reservoirs. J. Energy Resour. Technol. 2005, 130, 013301. [CrossRef]

14. Perkins, T.; Johnston, O. A Review of Diffusion and Dispersion in Porous Media. Soc. Pet. Eng. J. 1963, 3, 70-84. [CrossRef]

15. Coats, K.H.; Whitson, C.H.; Thomas, K. Modeling Conformance as Dispersion. SPE Reserv. Eval. Eng. 2009, 12, 33-47. [CrossRef]

16. Hughes, T.J.; Honari, A.; Graham, B.F.; Chauhan, A.S.; Johns, M.L.; May, E.F. $\mathrm{CO}_{2}$ sequestration for enhanced gas recovery: New measurements of supercritical $\mathrm{CO} 2-\mathrm{CH} 4$ dispersion in porous media and a review of recent research. Int. J. Greenh. Gas Control. 2012, 9, 457-468. [CrossRef]

17. Liu, S.; Zhang, Y.; Xing, W.; Jian, W.; Liu, Z.; Li, T.; Song, Y. Laboratory experiment of CO2-CH4 displacement and dispersion in sandpacks in enhanced gas recovery. J. Nat. Gas Sci. Eng. 2015, 26, 1585-1594. [CrossRef]

18. Honari, A.; Hughes, T.J.; Fridjonsson, E.O.; Johns, M.L.; May, E.F. Dispersion of supercritical CO2 and CH4 in consolidated porous media for enhanced gas recovery simulations. Int. J. Greenh. Gas Control 2013, 19, 234-242. [CrossRef]

19. Coats, K.H.; Whitson, C.H. SPE 90390 Modeling Conformance as Dispersion. In Proceedings of the SPE Annual Technical Conference and Exhibition, Houston, TX, USA, 26-29 September 2004.

20. Bjerg, P. Dispersion in Aquifers; DTU Environment: Lyngby, Denmark, 2008.

21. Schulze-Makuch, D. Longitudinal dispersivity data and implications for scaling behaviour. Groundwater 2005, 43, 443-456. [CrossRef] [PubMed]

(C) 2019 by the authors. Licensee MDPI, Basel, Switzerland. This article is an open access article distributed under the terms and conditions of the Creative Commons Attribution (CC BY) license (http://creativecommons.org/licenses/by/4.0/). 